\title{
Revinter
}

\section{Análise multifatorial de métodos volumétricos para a qualificação de ácidos ascórbico em comprimidos efervescentes}

Leonardo Tibiriçá Corrêa ${ }^{i}$

Giovanni Alves Munizi

Pedro Henrique Lopes Carafiniiii

Alexandre Bertolaccini Vergani ${ }^{\mathrm{iv}}$

Kauany de Araújo Ferreira ${ }^{v}$

Leandro Erico Batista ${ }^{\text {vi }}$

André Rinaldi Fukushima ${ }^{\text {vii }}$

Registro DOI: http://dx.doi.org/10.22280/revintervol11ed3.376

\section{Resumo}

Os comprimidos efervescentes tem sido uma forma farmacêutica das mais utilizadas para a reposição de ácido ascórbico, pois é uma forma rápida para obtenção do micronutrientes. $\mathrm{O}$ ácido ascórbico está é uma coenzima de diversas enzimas que realizam mecanismos metabólicos. Dentre as diversas funções que possui, uma delas é o estimulo o sistema imunológico. Sua falta gera a patologia denominada escorbuto, portanto, está diretamente relacionada a sua prevenção. Encontra-se presente principalmente em frutas cítricas e quase todas as verduras, é poderoso agente redutor, deve-se a essa característica um dos métodos baratos, rápidos, simples que pode ser utilizado para seu doseamento é a volumetria de oxirredução, mais especificamente, iodimetria para sua análise química quantitativa. As análises multifatoriais são importantes ferramentas para o desenvolvimento e validação de um método analítico quantitativo. São realizadas modificando determinadas figuras de mérito em um determinado método e avaliação estatística com a finalidade de se avaliar variação, significante ou não entre os valores experimentais. O presente trabalho tem como objetivo verificar se realmente há diferenças significativas frente a modificações no método, como por exemplo, concentração de solução padrão, procedência, volume de amostra, bem como demais figuras de mérito. As figuras de mérito avaliadas, apresentaram diferenças significantes para a análise multifatorial, mostrando a baixa robustez e reprodutibilidade para esse tipo de análise.

Palavras-chave: Ácido ascórbico. Análise multifatorial. Iodimetria. Análise estatística.

Multifactorial analysis of volumetric methods for the quantification of ascorbic acid in effervescent tablets

\section{Abstract}




\section{Revinter}

Effervescent tablets have been a widely used pharmaceutical form for the replacement of ascorbic acid, since it is a quick way to obtain micronutrients. Ascorbic acid is a coenzyme of several enzymes that perform metabolic mechanisms. Among the various functions it has, one of them is the stimulation of the immune system. Its lack generates the pathology called scurvy, so it is directly related to its prevention. It is present mainly in citrus fruits and almost all vegetables, it is powerful reducing agent, it is due to this characteristic one of the inexpensive, quick, simple methods that can be used for its dosing is oxidation volumetry, more specifically iodimetry for their quantitative chemical analysis. Multifactorial analyzes are important tools for the development and validation of a quantitative analytical method. They are performed by modifying certain figures of merit in a given method and statistical evaluation in order to evaluate variation, significant or not between experimental values. The present work aims to verify if there are actually significant differences with regard to modifications in the method, such as concentration of standard solution, origin, sample volume, as well as other figures of merit. The merit figures evaluated presented significant differences for the multifactorial analysis, showing the low robustness and reproducibility for this type of analysis.

Keywords: Ascorbic acid. Multifactorial analysis. Iodimetry. Statistical analysis.

\section{Recebido em 05/03/2018 Aceito em 02/10/2018}

\section{INTRODUÇÃO}

Vitaminas são biomoléculas com baixo peso molecular, atuam em no organismo, em pequenas doses não possuindo qualquer valor energético. Sua ingesta é imprescindivel uma vez que não são biossintetizadas de forma endógena, portanto, é necessário assegurar uma fonte exógena desses nutrientes (GUILLAND; LEQUEU, 1995).

A palavra vitamina é de etmologia "vital amine", foi criado por Casimir Funk, 1912, e alcunhava como sendo o "fator anti-beribéri”. Sua composição principal é de substâncias orgânicas complexas que naturalmente são produzidas em vegetais e alguns animais, principalmente exercendo papel no controle do metabolismo, com importância variada conforme a espécie (TEIXEIRA; NETO, 2009).

Classificam-se as vitaminas em hidrossolúveis: complexo $\mathrm{B}$, vitamina $\mathrm{C}$, ácido fólico e etc; e lipossolúveis: vitamina A, D, E e K. As vitaminas de maior importância que atuam como antioxidante são a vitamina A, E e C. São utilizadas para prevenção e tratamento de carência 


\section{Revinter}

nutricional e terapia de doenças não relacionadas à deficiência (FUCHS; WANNMACHER, 2010).

A ingestão de vitamina $\mathrm{C}$, também chamada de ácido ascórbico é indispensável para o organismo humano, que não a produz, entretanto, outros organismos possuem a capacidade de produzi-la endogenamente (TEXEIRA; NETO, 2009). Uma das alternativas para a reposição desse tipo de nutriente são as formulações efervescentes que possuem doses adequadas de reposição.

O primeiro Estudo de Doença Ocular Relacionado à Idade (AREDS) descobriu que a suplementação com vitamina $\mathrm{C}$, E, betacaroteno, zinco e cobre reduziu o risco do desenvolvimento da degeneração macular avançada (AMD) (AREDS, 2001).

Em 1928, Albert Szent-Gyorgy e em 1930, Glen King isolaram independentemente o ácido ascórbico. Sua fórmula foi determinada em 1933, quando foi sintetizada (Figura 1) (TEXEIRA; NETO, 2009).

\section{Figura 1: Moléculas de ácido ascórbico.}

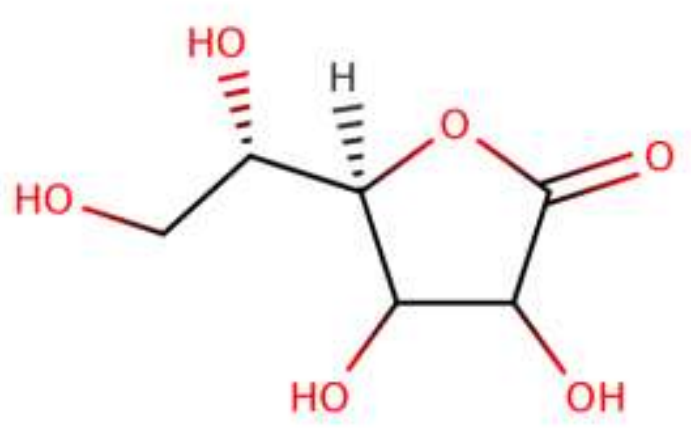

L-Ácido Ascorbico

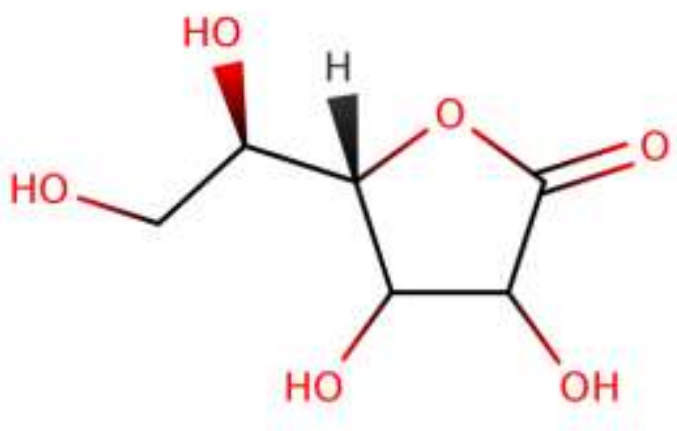

D-Ácido Ascorbico

Sua absorção é quase que totalmente no intestino delgado por processo ativo. É facilitada quando há a conversão de ácido ascórbico para ácido diidroascórbico. Pela circulação chega a tecidos renais, adrenais, hepáticos e esplênicos. Excreta-se na urina na forma de ácido oxálico, ácido treônico, L-xilose e ascorbato-2-sulfato, se ingerida até $100 \mathrm{~g} / \mathrm{dia}$. A ingestão acima desse valor, a excreção é feita pelos próprios rins (TEXEIRA; NETO, 2009).

A composição da maioria das formulações de ácido ascórbico $1 \mathrm{~g}$, encontram-se as seguintes interações: 


\section{Revinter}

- Com analgésicos, principalmente ácido acetilsalicílico, pois diminui o nível tecidual de ácido ascórbico;

- $\quad$ Com corticosteroides, pois aumentam o requerimento de ácido ascórbico;

- $\quad$ Com barbitúricos, pois aumentam a necessidade diária de ácido ascórbico;

- $\quad$ Com deferoxamina, pois potencializa os efeitos do ferro nos tecidos;

- $\quad$ E com salicilatos, pois podem aumentar a excreção de ácido ascórbico na urina.

É de grande importância para o organismo, pois participa das principais sínteses de proteína, elastina e colágeno. O consumo de alimentos rico em vitamina $\mathrm{C}$ combate os radicais livres, responsáveis pelas oxidações das células. Além do potencial antioxidante, também possui alta atuação no sistema imunológico, que, ao longo prazo, evita resfriados (EDUQUIM, 2012).

O ácido ascórbico atua na biossíntese de carnitina, síntese de neurotransmissores, reações de hidroxilação do colesterol, na absorção de ferro pelo intestino e promove estabilidade do ácido fólico e vitamina E (TEIXEIRA; NETO, 2009).

Sua ação antioxidante previne ou reduz os danos causados por oxidação de lipídios, proteínas e ácidos nucleicos causados por radicais livres, principalmente espécies de oxigênio reativo (COUTO; CANNIATTI-BRAZACA, 2010).

As recomendações dietéticas baseadas em idades são falhas, uma vez em que se tem variáveis como sexo, doenças, drogas, estado bioquímico e nutricional também. Para isso, recomenda-se uma dose diária de $10 \mathrm{mg}$ de ácido ascórbico, suficiente para prevenção do escorbuto (BLUMBERG, 1994; TEXEIRA; NETO, 2009).

Disponibiliza-se principalmente em frutas cítricas, mas todas as verduras e frutas possuem alguma quantidade de ácido ascórbico. Quanto mais fresco melhor para serem consumidos, pois o ácido ascórbico possui alta sensibilidade a diversas substâncias como zinco e pectina, a calor, variações de pH e luz (ESCOTT-STUMP; KRAUSE; MAHAN, 2010; RUSSO; SANTANA, 2013).

O ácido ascórbico possui alta poder oxidante, sendo então um ótimo agente redutor. Para isso, sua a forma mais comum para se dosear ácido ascórbico em comprimidos efervescentes é a volumetria de oxirredução.

Chama-se de reação de oxirredução quando há a transferência de elétrons, ou seja, reação de oxidação e redução. A reação de oxidação consiste na perda de elétrons e consequente Revinter, v. 11, n. 03, p. 54-73, out. 2018.

Página 57 | 73 


\section{Revinter}

aumento de seu número de oxidação. A reação de redução envolve o ganho de elétrons, portanto reduzindo seu número de oxidação (BACCAN et al., 1979).

Para a quantificação de ácido ascórbico é comumente envolvendo reações com iodo, podendo variar o método em iodometria - oxidação dos íons iodeto - ou iodimetria - redução do iodo (BACCAN et al., 1979).

A iodometria consiste na titulação entre o excesso de iodeto de potássio e uma solução padrão de tiossulfato de sódio, sendo que este deve ser previamente padronizado com solução de dicromato de potássio. O indicador utilizado deve ser o amido, mas deve ser somente adicionado quando estiver próximo do ponto final da titulação, uma vez que o iodo tende a ficar ligado ao amido após o ponto final, fornecendo resultados errôneos (BACCAN et al., 1979).

A iodimetria possui como solução padrão o iodo. $\mathrm{O}$ amido também é utilizado como indicador, mas neste caso deve ser adicionado no início da titulação. O ponto final é atingido quando a solução do erlenmeyer muda para a coloração azul escuro (BACCAN et al., 1979).

Ambos os métodos possuem fatores que afetam sua precisão e exatidão:

Tabela 1: Fatores que influenciam a iodometria e iodimetria

\begin{tabular}{cl}
\hline Fator & \multicolumn{1}{c}{ Consequência } \\
\hline Temperatura & $\begin{array}{l}\text { Altas temperaturas volatilizam o iodo e } \\
\text { afetam a utilização do amido }\end{array}$ \\
\hline $\mathrm{pH}>8,0$ & $\begin{array}{l}\text { A alta concentração de } \mathrm{OH}^{-} \text {reage com o iodo } \\
\text { e forma o íon hipoiodito }\left(\mathrm{IO}^{-}\right)^{3}, \text { um oxidante } \\
\text { muito mais forte que o próprio iodo }\end{array}$ \\
Volatilização & $\begin{array}{l}\text { O iodo é naturalmente volátil. Pode ser } \\
\text { evitado quando adicionado excesso de iodeto }\end{array}$ \\
& $\begin{array}{l}\text { Em meios ácidos, exposição à luz e exposição } \\
\text { prolongada pelo ar atmosférico, promove a } \\
\text { oxidação dos íons iodetos }\end{array}$ \\
\hline
\end{tabular}

\section{MATERIAIS E MÉTODOS}

\subsection{Materiais}

O preparo das soluções padrões e outros reagentes seguiu a norma NBR 11.589/89, com vidrarias recém-calibradas. Alguns reagentes foram comprados já padronizados e fatorados. 


\section{Revinter}

\section{Instrumentos e vidrarias}

Balança analítica (GEHAKA® AG200). Erlenmeyer de $500 \mathrm{~mL}$, bureta de $25 \mathrm{~mL}$, balões volumétricos de $1 \mathrm{~L}$ e $250 \mathrm{~mL}$, pipetas volumétricas de $1 \mathrm{~mL}, 2 \mathrm{~mL}, 5 \mathrm{~mL}$ e $10 \mathrm{~mL}$. Chapa de aquecimento. Frasco âmbar. Almofariz com pistilo.

\section{Reagentes}

Iodo $0,1 \mathrm{~N} ; 0,2 \mathrm{~N}$ e $\mathrm{Na}_{2} \mathrm{~S}_{2} \mathrm{O}_{3} \quad 0,1 \mathrm{~N}$ preparados no próprio laboratório, iodo $0,1 \mathrm{~N}$ e $\mathrm{Na}_{2} \mathrm{~S}_{2} \mathrm{O}_{3}$ 0,1 N (Synth®) e amido 0,5; 1,0 e 1,5\%. Padrão de ácido ascórbico de 0,5; 1,0; 1,2; 1,5; 1,8; 2,0 g/L. Placebo de vitamina $\mathrm{C}$ a fim da determinação de seletividade.

\section{Amostra utilizada}

Foram utilizadas duas marcas com maior índice de vendas nas drogarias da região. São comprimidos efervescentes com $1 \mathrm{~g}$ de vitamina $\mathrm{C}$ por comprimido sem açúcar. A amostra foi dissolvida em balão de 1 L e armazenada em frasco âmbar. Todo dia de execução foi preparada uma nova solução, já que esta apresenta diversas sensibilidades e facilidade para degradação.

\subsection{Metodologia}

\section{Original}

O método originalmente consistia na iodimetria, ou seja, titulação entre a solução padrão de iodo $0,1 \mathrm{~N}, 1 \mathrm{~mL}$ de amostra e $5 \mathrm{~mL}$ de amido $1 \%$. Titula-se até o surgimento da cor azul escura como ponto final do método.

A seguinte reação mostra o ponto de equivalência do método:

$\mathrm{C}_{6} \mathrm{H}_{8} \mathrm{O}_{6}+\mathrm{I}_{2} \rightarrow 2 \mathrm{HI}+\mathrm{C}_{6} \mathrm{H}_{6} \mathrm{O}_{6}$

$\mathrm{C}_{6} \mathrm{H}_{8} \mathrm{O}_{6}+\mathrm{I}_{2} \rightarrow 2 \mathrm{I}^{-}+2 \mathrm{H}^{+}+\mathrm{C}_{6} \mathrm{H}_{6} \mathrm{O}_{6}$

\section{Variações}

1. Utilização de $\mathrm{Na}_{2} \mathrm{~S}_{2} \mathrm{O}_{3}$ 0,1 $\mathrm{N}$ como solução padrão (iodometria);

2. Utilização de Iodo $0,1 \mathrm{~N}$ de outra procedência (Synt®);

3. Utilização de $\mathrm{Na}_{2} \mathrm{~S}_{2} \mathrm{O}_{3} 0,1 \mathrm{~N}$ de outra procedência (Synt $\left.{ }^{\circledR}\right)$;

4. Utilização de Iodo $0,2 \mathrm{~N}$

5. Utilização de amido $1,5 \%$;

6. Utilização de amido $0,5 \%$

7. Variação no volume de amostra entre 1, 2, 5 e $10 \mathrm{~mL}$;

8. Variação de analista; 
9. Variação de marcas;

10. Variação de concentração de padrão (linearidade);

11. Avaliação da seletividade.

\section{RESULTADOS}

Comparação entre amostras:

Tabela 2: Análise estatística comparativa entre amostras.

\begin{tabular}{ccccccc}
\hline Amostra & Média & $\begin{array}{c}\text { Desvio } \\
\text { Padrão }\end{array}$ & $\begin{array}{c}\text { Erro padrão } \\
\text { da média }\end{array}$ & $\begin{array}{c}\text { Coeficiente } \\
\text { de variação }\end{array}$ & $\begin{array}{c}\text { Erro } \\
\text { relativo }\end{array}$ & Teste t \\
\hline A & $2,3776 \mathrm{~g}$ & $1,4467 \mathrm{~g}$ & $0,4575 \mathrm{~g}$ & $60,85 \%$ & $137,76 \%$ & \multirow{2}{*}{1,512} \\
\hline B & $0,8860 \mathrm{~g}$ & $0,0000 \mathrm{~g}$ & $0,0000 \mathrm{~g}$ & $0,00 \%$ & $-11,94 \%$ & \\
\hline
\end{tabular}

Tabela 3: Tabela ANOVA comparativa entre valores experimentais para duas amostras diferentes.

\begin{tabular}{cccccc}
\hline $\begin{array}{c}\text { Fonte de } \\
\text { variação }\end{array}$ & $\begin{array}{c}\text { Soma dos } \\
\text { Quadrados }\end{array}$ & $\begin{array}{c}\text { Graus de } \\
\text { Liberdade }\end{array}$ & $\begin{array}{c}\text { Quadrado } \\
\text { Médio }\end{array}$ & F & p - Valor \\
\hline $\begin{array}{c}\text { Entre os } \\
\text { grupos }\end{array}$ & 11,2050 & 1 & 11,2050 & & \\
$\begin{array}{c}\text { Dentro dos } \\
\text { grupos }\end{array}$ & 18,8365 & 18 & 0,9556 & 11,726 & $7,9 \times 10^{-5}$ \\
\hline TOTAL & 30,0415 & 19 & 12,1606 & & \\
\hline
\end{tabular}

Modificação no volume pipetado de amostra A:

Tabela 4: Comparação de resultados para diferentes volumes de amostra A.

\begin{tabular}{cccccc}
\hline $\begin{array}{c}\text { Volume de } \\
\text { amostra }\end{array}$ & Média & $\begin{array}{c}\text { Desvio } \\
\text { Padrão }\end{array}$ & $\begin{array}{c}\text { Erro } \\
\text { padrão } \\
\text { da média }\end{array}$ & $\begin{array}{c}\text { Coeficiente } \\
\text { de variação }\end{array}$ & $\begin{array}{c}\text { Erro } \\
\text { relativo }\end{array}$ \\
\hline $\mathbf{1} \mathbf{~ m L}$ & $2,3776 \mathrm{~g}$ & $0,9329 \mathrm{~g}$ & $0,2950 \mathrm{~g}$ & $39,24 \%$ & $137,76 \%$ \\
$\mathbf{2} \mathbf{~ m L}$ & $2,0254 \mathrm{~g}$ & $0,4254 \mathrm{~g}$ & $0,1345 \mathrm{~g}$ & $21 \%$ & $102,54 \%$ \\
$\mathbf{5} \mathbf{~} \mathbf{L}$ & $1,1624 \mathrm{~g}$ & $0,0909 \mathrm{~g}$ & $0,0288 \mathrm{~g}$ & $7,82 \%$ & $16,24 \%$ \\
$\mathbf{1 0} \mathbf{~ m L}$ & $1,0479 \mathrm{~g}$ & $0,0278 \mathrm{~g}$ & $0,0088 \mathrm{~g}$ & $2,66 \%$ & $4,79 \%$ \\
\hline
\end{tabular}


Tabela 5: Tabela ANOVA comparativa entre os valores experimentais para diferentes volumes de amostra $A$.

\begin{tabular}{cccccc}
\hline $\begin{array}{c}\text { Fonte de } \\
\text { variação }\end{array}$ & $\begin{array}{c}\text { Soma dos } \\
\text { Quadrados }\end{array}$ & $\begin{array}{c}\text { Graus de } \\
\text { Liberdade }\end{array}$ & $\begin{array}{c}\text { Quadrado } \\
\text { Médio }\end{array}$ & F & p-valor \\
\hline $\begin{array}{c}\text { Entre os } \\
\text { grupos }\end{array}$ & 12,7058 & 3 & 4,2353 & 1,0922 & $9,08 \times 10^{-7}$ \\
$\begin{array}{c}\text { Dentro dos } \\
\text { grupos }\end{array}$ & 9,5420 & 36 & 3,7728 & & \\
\hline TOTAL & 22,2478 & 39 & 8,1129 & & \\
\hline
\end{tabular}

Modificação no volume pipatado de amostra B:

Tabela 6: Análise estatística comparativa entre diferentes volumes de amostra B.

\begin{tabular}{cccccc}
\hline $\begin{array}{c}\text { Volume } \\
\text { de } \\
\text { amostra }\end{array}$ & Média & $\begin{array}{c}\text { Desvio } \\
\text { Padrão }\end{array}$ & $\begin{array}{c}\text { Erro } \\
\text { padrão da } \\
\text { média }\end{array}$ & $\begin{array}{c}\text { Coeficiente } \\
\text { de variação }\end{array}$ & $\begin{array}{c}\text { Erro } \\
\text { relativo }\end{array}$ \\
\hline $\mathbf{1} \mathbf{~ m L}$ & $0,8806 \mathrm{~g}$ & $0,0000 \mathrm{~g}$ & $0,0000 \mathrm{~g}$ & $0,00 \%$ & $-11,94 \%$ \\
$\mathbf{2} \mathbf{~ m L}$ & $1,8493 \mathrm{~g}$ & $0,3827 \mathrm{~g}$ & $0,1210 \mathrm{~g}$ & $20,70 \%$ & $84,93 \%$ \\
$\mathbf{5} \mathbf{~ m L}$ & $1,2152 \mathrm{~g}$ & $0,1542 \mathrm{~g}$ & $0,0488 \mathrm{~g}$ & $12,69 \%$ & $21,52 \%$ \\
$\mathbf{1 0} \mathbf{~ m L}$ & $1,0215 \mathrm{~g}$ & $0,0742 \mathrm{~g}$ & $0,0235 \mathrm{~g}$ & $7,27 \%$ & $2,15 \%$ \\
\hline
\end{tabular}

Tabela 7: Tabela ANOVA comparativa entre valores experimentais de diferentes volumes de amostra $B$.

\begin{tabular}{cccccc}
\hline $\begin{array}{c}\text { Fonte de } \\
\text { variação }\end{array}$ & $\begin{array}{c}\text { Soma dos } \\
\text { Quadrados }\end{array}$ & $\begin{array}{c}\text { Graus de } \\
\text { Liberdade }\end{array}$ & $\begin{array}{c}\text { Quadrado } \\
\text { Médio }\end{array}$ & F & p - valor \\
\hline $\begin{array}{c}\text { Entre os } \\
\text { grupos }\end{array}$ & 5,4870 & 3 & 1,8290 & & \\
$\begin{array}{c}\text { Dentro dos } \\
\text { grupos }\end{array}$ & 1,5819 & 36 & 0,0439 & 61,6233 & $6,02 \times 10^{-15}$ \\
\hline TOTAL & 7,0689 & 39 & 1,8730 & & \\
\hline Fonte: Corrêa & & & &
\end{tabular}

Fonte: Corrêa, 2017.

Modificação na concentração de amido com a amostra A: 
Tabela 8: Análise estatística comparativa entre diferentes concentrações de amido para quantificação de amostra $A$.

\begin{tabular}{ccccc}
\hline Amido & Média & $\begin{array}{c}\text { Desvio } \\
\text { Padrão }\end{array}$ & $\begin{array}{c}\text { Erro } \\
\text { padrão da } \\
\text { média }\end{array}$ & $\begin{array}{c}\text { Coeficiente } \\
\text { de variação }\end{array}$ \\
\hline $\mathbf{0 , 5 \%}$ & $2,2896 \mathrm{~g}$ & $0,8507 \mathrm{~g}$ & $0,2690 \mathrm{~g}$ & $37,16 \%$ \\
$\mathbf{1 , 0 \%}$ & $2,3776 \mathrm{~g}$ & $0,9329 \mathrm{~g}$ & $0,2950 \mathrm{~g}$ & $39,23 \%$ \\
$\mathbf{1 , 5 \%}$ & $2,2015 \mathrm{~g}$ & $0,4641 \mathrm{~g}$ & $0,1468 \mathrm{~g}$ & $21,08 \%$ \\
\hline
\end{tabular}

Tabela 9: Tabela ANOVA comparativa entre diferentes concentrações de amido para quantificação de amostra $A$.

\begin{tabular}{cccccc}
\hline $\begin{array}{c}\text { Fonte de } \\
\text { variação }\end{array}$ & $\begin{array}{c}\text { Soma dos } \\
\text { Quadrados }\end{array}$ & $\begin{array}{c}\text { Graus de } \\
\text { Liberdade }\end{array}$ & $\begin{array}{c}\text { Quadrado } \\
\text { Médio }\end{array}$ & F & p-valor \\
\hline $\begin{array}{c}\text { Entre os } \\
\text { grupos }\end{array}$ & 0,1551 & 2 & 0,077546 & & \\
$\begin{array}{c}\text { Dentro dos } \\
\text { grupos }\end{array}$ & 16,2846 & 27 & 0,603133 & 0,1286 & 0,8799 \\
\hline TOTAL & 16,4397 & 29 & 0,680678 & & \\
\hline
\end{tabular}

Modificação na concentração de amido com a amostra B:

Tabela 10: Análise comparativa de resultados entre diferentes concentrações de amido para quantificação de amostra $B$.

\begin{tabular}{ccccc}
\hline Amido & Média & $\begin{array}{c}\text { Desvio } \\
\text { Padrão }\end{array}$ & $\begin{array}{c}\text { Erro } \\
\text { padrão da } \\
\text { média }\end{array}$ & $\begin{array}{c}\text { Coeficiente } \\
\text { de variação }\end{array}$ \\
\hline $\mathbf{0 , 5 \%}$ & $1,6371 \mathrm{~g}$ & $0,2785 \mathrm{~g}$ & $0,0880 \mathrm{~g}$ & $16,64 \%$ \\
$\mathbf{1 , 0 \%}$ & $0,8860 \mathrm{~g}$ & $0,0000 \mathrm{~g}$ & $0,0000 \mathrm{~g}$ & $0,00 \%$ \\
$\mathbf{1 , 5 \%}$ & $1,7612 \mathrm{~g}$ & $0,0000 \mathrm{~g}$ & $0,0000 \mathrm{~g}$ & $0,00 \%$ \\
\hline
\end{tabular}

Tabela 11: Tabela ANOVA comparativa entre diferentes concentrações de amido para quantificação de amostra $B$.

\begin{tabular}{cccccc}
\hline $\begin{array}{c}\text { Fonte de } \\
\text { variação }\end{array}$ & $\begin{array}{c}\text { Soma dos } \\
\text { Quadrados }\end{array}$ & $\begin{array}{c}\text { Graus de } \\
\text { Liberdade }\end{array}$ & $\begin{array}{c}\text { Quadrado } \\
\text { Médio }\end{array}$ & F & p-valor \\
\hline $\begin{array}{c}\text { Entre os } \\
\text { grupos }\end{array}$ & 4,7044 & 2 & 2,3522 & & \\
$\begin{array}{c}\text { Dentro dos } \\
\text { grupos }\end{array}$ & 0,6979 & 27 & 0,0258 & 91,0000 & $1,003 \times 10^{-12}$ \\
\hline TOTAL & 5,4023 & 29 & 2,3781 & & \\
\hline
\end{tabular}

Revinter, v. 11, n. 03, p. 54-73, out. 2018. 


\section{Iodometria com amostra A:}

Tabela 12: Análise estatística entre resultados obtidos para diferentes métodos de quantificação de amostra $A$.

\begin{tabular}{lcccccc}
\hline Método & Média & $\begin{array}{c}\text { Desvio } \\
\text { Padrão }\end{array}$ & $\begin{array}{c}\text { Erro } \\
\text { padrão da } \\
\text { média }\end{array}$ & $\begin{array}{c}\text { Coeficiente } \\
\text { de variação }\end{array}$ & $\begin{array}{c}\text { Erro } \\
\text { relativo }\end{array}$ & Teste t \\
\hline Iodimetria & $2,3776 \mathrm{~g}$ & $0,9329 \mathrm{~g}$ & $0,2950 \mathrm{~g}$ & $39,24 \%$ & $137,76 \%$ & \multirow{2}{*}{3,488} \\
\hline Iodometria & $1,2328 \mathrm{~g}$ & $0,4547 \mathrm{~g}$ & $0,1438 \mathrm{~g}$ & $36,86 \%$ & $23,28 \%$ & \\
\hline
\end{tabular}

Tabela 13: Tabela ANOVA comparativa entre valores experimentais para diferentes métodos de quantificação de amostra $A$.

\begin{tabular}{cccccc}
\hline $\begin{array}{c}\text { Fonte de } \\
\text { variação }\end{array}$ & $\begin{array}{c}\text { Soma dos } \\
\text { Quadrados }\end{array}$ & $\begin{array}{c}\text { Graus de } \\
\text { Liberdade }\end{array}$ & $\begin{array}{c}\text { Quadrado } \\
\text { Médio }\end{array}$ & F & p - valor \\
\hline $\begin{array}{c}\text { Entre os } \\
\text { grupos }\end{array}$ & 6,5526 & 1 & 6,5526 & & \\
$\begin{array}{c}\text { Dentro dos } \\
\text { grupos }\end{array}$ & 9,6939 & 18 & 0,5385 & 12,1672 & 0,002624 \\
\hline TOTAL & 16,2464 & 19 & 7,0912 & & \\
\hline
\end{tabular}

Iodometria com amostra B:

Tabela 14: Análise estatística entre resultados de diferentes métodos para quantificação de amostra $B$.

\begin{tabular}{lcccccc}
\hline Método & Média & $\begin{array}{c}\text { Desvio } \\
\text { Padrão }\end{array}$ & $\begin{array}{c}\text { Erro } \\
\text { padrão da } \\
\text { média }\end{array}$ & $\begin{array}{c}\text { Coeficiente } \\
\text { de variação }\end{array}$ & $\begin{array}{c}\text { Erro } \\
\text { relativo }\end{array}$ & Teste t \\
\hline Iodimetria & $0,8806 \mathrm{~g}$ & $0,0000 \mathrm{~g}$ & $0,0000 \mathrm{~g}$ & $0,00 \%$ & $-11,94 \%$ & $-3,00$ \\
\hline Iodometria & $1,3029 \mathrm{~g}$ & $0,4641 \mathrm{~g}$ & $0,1468 \mathrm{~g}$ & $35,14 \%$ & $32,09 \%$ & - \\
\hline
\end{tabular}

Tabela 15: Tabela ANOVA entre valores experimentais para diferentes métodos de quantificação de amostra $B$.

\begin{tabular}{cccccc}
\hline $\begin{array}{c}\text { Fonte de } \\
\text { variação }\end{array}$ & $\begin{array}{c}\text { Soma dos } \\
\text { Quadrados }\end{array}$ & $\begin{array}{c}\text { Graus de } \\
\text { Liberdade }\end{array}$ & $\begin{array}{c}\text { Quadrado } \\
\text { Médio }\end{array}$ & F & p-valor \\
\hline $\begin{array}{c}\text { Entre os } \\
\text { grupos }\end{array}$ & 0,9693 & 1 & 0,9693 & 9,0000 & 0,0077 \\
\hline
\end{tabular}




\begin{tabular}{cccc}
\hline $\begin{array}{c}\text { Dentro dos } \\
\text { grupos }\end{array}$ & 1,9386 & 18 & 0,1077 \\
\hline TOTAL & 2,9080 & 19 & 1,0770 \\
\hline
\end{tabular}

Variação na concentração do iodo amostra A:

Tabela 16: Análise estatística entre resultados obtidos perante variação da concentração de iodo com amostra $A$.

\begin{tabular}{ccccccc}
\hline Iodo & Média & $\begin{array}{c}\text { Desvio } \\
\text { Padrão }\end{array}$ & $\begin{array}{c}\text { Erro padrão } \\
\text { da média }\end{array}$ & $\begin{array}{c}\text { Coeficiente } \\
\text { de variação }\end{array}$ & $\begin{array}{c}\text { Erro } \\
\text { relativo }\end{array}$ & Teste t \\
\hline $\mathbf{0 , 1 N}$ & $2,3776 \mathrm{~g}$ & $0,9329 \mathrm{~g}$ & $0,2950 \mathrm{~g}$ & $39,24 \%$ & $137, \%$ & 2,089 \\
$\mathbf{0 , 2 N}$ & $1,7612 \mathrm{~g}$ & $0,0000 \mathrm{~g}$ & $0,0000 \mathrm{~g}$ & $0,00 \%$ & $76,12 \%$ & 2,089 \\
\hline
\end{tabular}

Tabela 17: Tabela ANOVA comparativo entre valores experimentais perante a variação da concentração de iodo com amostra $A$.

\begin{tabular}{cccccc}
\hline $\begin{array}{c}\text { Fonte de } \\
\text { variação }\end{array}$ & $\begin{array}{c}\text { Soma dos } \\
\text { Quadrados }\end{array}$ & $\begin{array}{c}\text { Graus de } \\
\text { Liberdade }\end{array}$ & $\begin{array}{c}\text { Quadrado } \\
\text { Médio }\end{array}$ & F & p-valor \\
\hline $\begin{array}{c}\text { Entre os } \\
\text { grupos }\end{array}$ & 1,8997 & 1 & 1,8997 & & \\
$\begin{array}{c}\text { Dentro dos } \\
\text { grupos }\end{array}$ & 7,8327 & 18 & 2,2981 & 0,8267 & 0,0511 \\
\hline TOTAL & 9,7325 & 19 & 4,1978 & & \\
\hline
\end{tabular}

Variação na concentração do iodo amostra B:

Tabela 18: Análise estatística entre resultados obtidos perante a variação de concentração de iodo com amostra $B$.

\begin{tabular}{ccccccc}
\hline Iodo & Média & $\begin{array}{c}\text { Desvio } \\
\text { Padrão }\end{array}$ & $\begin{array}{c}\text { Erro padrão } \\
\text { da média }\end{array}$ & $\begin{array}{c}\text { Coeficiente } \\
\text { de variação }\end{array}$ & $\begin{array}{c}\text { Erro } \\
\text { relativo }\end{array}$ & Teste t \\
\hline $\mathbf{0 , 1 N}$ & $0,8806 \mathrm{~g}$ & $0,0000 \mathrm{~g}$ & $0,0000 \mathrm{~g}$ & $0,00 \%$ & $-11,94 \%$ & $-6,00$ \\
$\mathbf{0 , 2 N}$ & $1,9373 \mathrm{~g}$ & $0,5569 \mathrm{~g}$ & $0,1761 \mathrm{~g}$ & $28,75 \%$ & $93,73 \%$ & -6 \\
\hline
\end{tabular}


Tabela 19: Tabela ANOVA comparativa entre valores experimentais perante a variação de concentração de iodo com amostra $B$.

\begin{tabular}{cccccc}
\hline $\begin{array}{c}\text { Fonte de } \\
\text { variação }\end{array}$ & $\begin{array}{c}\text { Soma dos } \\
\text { Quadrados }\end{array}$ & $\begin{array}{c}\text { Graus de } \\
\text { Liberdade }\end{array}$ & $\begin{array}{c}\text { Quadrado } \\
\text { Médio }\end{array}$ & F & p-valor \\
\hline $\begin{array}{c}\text { Entre os } \\
\text { grupos }\end{array}$ & 5,5833 & 1 & 5,5833 & & \\
$\begin{array}{c}\text { Dentro dos } \\
\text { grupos }\end{array}$ & 2,7916 & 18 & 6,4478 & 0,8659 & $1,2 \times 10^{-6}$ \\
\hline TOTAL & 8,3749 & 19 & 12,0311 & & \\
\hline
\end{tabular}

Comparação com iodo de outra procedência com amostra A:

Tabela 20: Análise estatística entre resultados obtidos com solução de iodo de diferentes procedências com amostra $A$.

\begin{tabular}{cccccc}
\hline \multicolumn{1}{c}{ Iodo } & Média & $\begin{array}{c}\text { Desvio } \\
\text { Padrão }\end{array}$ & $\begin{array}{c}\text { Erro padrão } \\
\text { da média }\end{array}$ & $\begin{array}{c}\text { Coeficiente de } \\
\text { variação }\end{array}$ & Teste t \\
\hline Laboratório & $2,3776 \mathrm{~g}$ & $0,9329 \mathrm{~g}$ & $0,2950 \mathrm{~g}$ & $39,24 \%$ & $-6,17 \times 10^{-5}$ \\
\hline LabSynth ${ }^{\circledR}$ & $2,3776 \mathrm{~g}$ & $0,4254 \mathrm{~g}$ & $0,1345 \mathrm{~g}$ & $5,66 \%$ & \\
\hline
\end{tabular}

Tabela 21: Tabela ANOVA comparativa entre valores experimentais obtidos com solução de iodo de diferentes procedências com amostra $A$.

\begin{tabular}{cccccc}
\hline $\begin{array}{c}\text { Fonte de } \\
\text { variação }\end{array}$ & $\begin{array}{c}\text { Soma dos } \\
\text { Quadrados }\end{array}$ & $\begin{array}{c}\text { Graus de } \\
\text { Liberdade }\end{array}$ & $\begin{array}{c}\text { Quadrado } \\
\text { Médio }\end{array}$ & F & p-valor \\
\hline $\begin{array}{c}\text { Entre os } \\
\text { grupos }\end{array}$ & $4 \times 10^{-9}$ & 1 & $4 \times 10^{-9}$ & & \\
$\begin{array}{c}\text { Dentro dos } \\
\text { grupos }\end{array}$ & 9,4612 & 18 & 1,9025 & $2,1 \times 10^{-9}$ & 0,00 \\
\hline TOTAL & 9,4612 & 19 & 1,9025 & & \\
\hline
\end{tabular}

Comparação com iodo de outra procedência com amostra B: 
Tabela 22: Análise estatística entre resultados obtidos com solução de iodo de diferentes procedências com amostra $B$.

\begin{tabular}{cccccc}
\hline \multicolumn{1}{c}{ Iodo } & Média & $\begin{array}{c}\text { Desvio } \\
\text { Padrão }\end{array}$ & $\begin{array}{c}\text { Erro padrão } \\
\text { da média }\end{array}$ & $\begin{array}{c}\text { Coeficiente de } \\
\text { variação }\end{array}$ & Teste t \\
\hline Laboratório & $0,8806 \mathrm{~g}$ & $0,0000 \mathrm{~g}$ & $0,0000 \mathrm{~g}$ & $0,00 \%$ & $-8,510$ \\
\hline LabSynth ${ }^{\circledR}$ & $2,0254 \mathrm{~g}$ & $0,4254 \mathrm{~g}$ & $0,1345 \mathrm{~g}$ & $6,64 \%$ & - \\
\hline
\end{tabular}

Tabela 23: Tabela ANOVA comparativa entre valores experimentais obtidos com solução de iodo de diferentes procedências com amostra $B$.

\begin{tabular}{cccccc}
\hline $\begin{array}{c}\text { Fonte de } \\
\text { variação }\end{array}$ & $\begin{array}{c}\text { Soma dos } \\
\text { Quadrados }\end{array}$ & $\begin{array}{c}\text { Graus de } \\
\text { Liberdade }\end{array}$ & $\begin{array}{c}\text { Quadrado } \\
\text { Médio }\end{array}$ & F & p - valor \\
\hline $\begin{array}{c}\text { Entre os } \\
\text { grupos }\end{array}$ & 6,9016 & 1 & 6,9016 & & \\
$\begin{array}{c}\text { Dentro dos } \\
\text { grupos }\end{array}$ & 1,6285 & 18 & 0,0905 & 76,2857 & $1 \times 10^{-7}$ \\
\hline TOTAL & 8,5300 & 19 & 6,9920 & & \\
\hline
\end{tabular}

Comparação com a iodometria com solução padrão de outra procedência na amostra A:

Tabela 24: Análise estatística entre diferentes procedências de solução padrão para a iodometria com amostra A.

\begin{tabular}{ccccccc}
\hline \multicolumn{1}{c}{ Iodo } & Média & $\begin{array}{c}\text { Desvio } \\
\text { Padrão }\end{array}$ & $\begin{array}{c}\text { Erro padrão } \\
\text { da média }\end{array}$ & $\begin{array}{c}\text { Coeficiente } \\
\text { de variação }\end{array}$ & $\begin{array}{c}\text { Erro } \\
\text { relativo }\end{array}$ & Teste t \\
\hline Laboratório & $1,2328 \mathrm{~g}$ & $0,4547 \mathrm{~g}$ & $0,1438 \mathrm{~g}$ & $36,86 \%$ & $23,28 \%$ & $-1,342$ \\
\hline LabSynth $\AA$ & $1,4970 \mathrm{~g}$ & $0,4254 \mathrm{~g}$ & $0,1345 \mathrm{~g}$ & $28,41 \%$ & $49,70 \%$ & $-1,34$ \\
\hline
\end{tabular}

Tabela 25: Tabela ANOVA comparativa entre valores experimentais entre diferentes procedências de solução padrão para a iodometria com amostra $A$.

\begin{tabular}{cccccc}
\hline $\begin{array}{c}\text { Fonte de } \\
\text { variação }\end{array}$ & $\begin{array}{c}\text { Soma dos } \\
\text { Quadrados }\end{array}$ & $\begin{array}{c}\text { Graus de } \\
\text { Liberdade }\end{array}$ & $\begin{array}{c}\text { Quadrado } \\
\text { Médio }\end{array}$ & F & p-valor \\
\hline $\begin{array}{c}\text { Entre os } \\
\text { grupos }\end{array}$ & 0,3491 & 1 & 0,3491 & & \\
$\begin{array}{c}\text { Dentro dos } \\
\text { grupos }\end{array}$ & 3,4892 & 18 & 0,1938 & 1,8007 & 0,1964 \\
\hline TOTAL & 3,8383 & 19 & 0,5429 & & \\
\hline
\end{tabular}


Comparação com a iodometria com solução padrão de outra procedência na amostra $B:$

Tabela 26: Análise estatística entre diferentes procedências de solução padrão para a iodometria com amostra $B$.

\begin{tabular}{ccccccc}
\hline \multicolumn{1}{c}{ Iodo } & Média & $\begin{array}{c}\text { Desvio } \\
\text { Padrão }\end{array}$ & $\begin{array}{c}\text { Erro padrão } \\
\text { da média }\end{array}$ & $\begin{array}{c}\text { Coeficiente } \\
\text { de variação }\end{array}$ & $\begin{array}{c}\text { Erro } \\
\text { relativo }\end{array}$ & Teste t \\
\hline Laboratório & $1,3209 \mathrm{~g}$ & $0,4641 \mathrm{~g}$ & $0,1468 \mathrm{~g}$ & $35,41 \%$ & $32,09 \%$ & $-1,362$ \\
\hline LabSynth $\AA$ & $1,5851 \mathrm{~g}$ & $0,3713 \mathrm{~g}$ & $0,1174 \mathrm{~g}$ & $23,42 \%$ & $58,51 \%$ & $-1,362$ \\
\hline
\end{tabular}

Tabela 27: Tabela ANOVA comparativa entre valores experimentais entre diferentes procedências de solução padrão para a iodometria com amostra $B$.

\begin{tabular}{cccccc}
\hline $\begin{array}{c}\text { Fonte de } \\
\text { variação }\end{array}$ & $\begin{array}{c}\text { Soma dos } \\
\text { Quadrados }\end{array}$ & $\begin{array}{c}\text { Graus de } \\
\text { Liberdade }\end{array}$ & $\begin{array}{c}\text { Quadrado } \\
\text { Médio }\end{array}$ & F & p-valor \\
\hline $\begin{array}{c}\text { Entre os } \\
\text { grupos }\end{array}$ & 0,3279 & 1 & 0,3279 & & \\
$\begin{array}{c}\text { Dentro dos } \\
\text { grupos }\end{array}$ & 3,1792 & 18 & 0,1766 & 1,856 & 0,1769 \\
\hline TOTAL & 3,5071 & 19 & 0,5045 & & \\
\hline
\end{tabular}

Comparação entre analista com amostra A:

Tabela 28: Análise estatística entre diferentes analista com amostra A.

\begin{tabular}{ccccc}
\hline Analista & Média & $\begin{array}{c}\text { Desvio } \\
\text { Padrão }\end{array}$ & $\begin{array}{c}\text { Erro padrão } \\
\text { da média }\end{array}$ & $\begin{array}{c}\text { Coeficiente de } \\
\text { variação }\end{array}$ \\
\hline $\mathbf{1}$ & $1,7297 \mathrm{~g}$ & $0,3472 \mathrm{~g}$ & $0,1098 \mathrm{~g}$ & $20,07 \%$ \\
$\mathbf{2}$ & $1,2797 \mathrm{~g}$ & $0,3472 \mathrm{~g}$ & $0,1098 \mathrm{~g}$ & $20,07 \%$ \\
$\mathbf{3}$ & $1,2797 \mathrm{~g}$ & $0,3472 \mathrm{~g}$ & $0,1098 \mathrm{~g}$ & $20,07 \%$ \\
$\mathbf{4}$ & $1,4970 \mathrm{~g}$ & $0,4254 \mathrm{~g}$ & $0,1345 \mathrm{~g}$ & $28,41 \%$ \\
$\mathbf{5}$ & $1,7612 \mathrm{~g}$ & $0,0000 \mathrm{~g}$ & $0,0000 \mathrm{~g}$ & $0,00 \%$ \\
\hline
\end{tabular}

Tabela 29: Tabela ANOVA comparativa entre valores experimentais de diferentes analistas com amostra $\mathrm{A}$.

\begin{tabular}{cccccc}
\hline $\begin{array}{c}\text { Fonte de } \\
\text { variação }\end{array}$ & $\begin{array}{c}\text { Soma dos } \\
\text { Quadrados }\end{array}$ & $\begin{array}{c}\text { Graus de } \\
\text { Liberdade }\end{array}$ & $\begin{array}{c}\text { Quadrado } \\
\text { Médio }\end{array}$ & F & p-valor \\
\hline $\begin{array}{c}\text { Entre os } \\
\text { grupos }\end{array}$ & 0,47025 & 4 & 0,118 & 1,083 & 0,367 \\
\hline
\end{tabular}




\begin{tabular}{cccc}
\hline $\begin{array}{c}\text { Dentro dos } \\
\text { grupos }\end{array}$ & 4,88298 & 45 & 0,109 \\
\hline TOTAL & 5,35323 & 49 & 0,226 \\
\hline
\end{tabular}

Comparação entre analista com amostra B:

Tabela 30: Análise estatística entre diferentes analistas com amostra B.

\begin{tabular}{ccccc}
\hline Analista & Média & $\begin{array}{c}\text { Desvio } \\
\text { Padrão }\end{array}$ & $\begin{array}{c}\text { Erro padrão } \\
\text { da média }\end{array}$ & $\begin{array}{c}\text { Coeficiente de } \\
\text { variação }\end{array}$ \\
\hline $\mathbf{1}$ & $0,8806 \mathrm{~g}$ & $0,0000 \mathrm{~g}$ & $0,0000 \mathrm{~g}$ & $0,00 \%$ \\
$\mathbf{2}$ & $0,8806 \mathrm{~g}$ & $0,0000 \mathrm{~g}$ & $0,0000 \mathrm{~g}$ & $0,00 \%$ \\
$\mathbf{3}$ & $0,9687 \mathrm{~g}$ & $0,2785 \mathrm{~g}$ & $0,0881 \mathrm{~g}$ & $28,75 \%$ \\
$\mathbf{4}$ & $1,3209 \mathrm{~g}$ & $0,4641 \mathrm{~g}$ & $0,1468 \mathrm{~g}$ & $35,14 \%$ \\
$\mathbf{5}$ & $0,8806 \mathrm{~g}$ & $0,0000 \mathrm{~g}$ & $0,0000 \mathrm{~g}$ & $0,00 \%$ \\
\hline
\end{tabular}

Tabela 31: Tabela ANOVA comparativa entre valores experimentais entre diferentes analistas com amostra $B$.

\begin{tabular}{cccccc}
\hline $\begin{array}{c}\text { Fonte de } \\
\text { variação }\end{array}$ & $\begin{array}{c}\text { Soma dos } \\
\text { Quadrados }\end{array}$ & $\begin{array}{c}\text { Graus de } \\
\text { Liberdade }\end{array}$ & $\begin{array}{c}\text { Quadrado } \\
\text { Médio }\end{array}$ & F & p-valor \\
\hline $\begin{array}{c}\text { Entre os } \\
\text { grupos }\end{array}$ & 1,4579 & 4 & 0,3645 & & \\
$\begin{array}{c}\text { Dentro dos } \\
\text { grupos }\end{array}$ & 2,6366 & 45 & 0,0586 & 0,221 & 0005 \\
\hline TOTAL & 4,0944 & 49 & 0,4231 & & \\
\hline
\end{tabular}

Comparação com diferentes concentrações de padrão: 
Figura 2: Representação gráfica de diferentes concentrações de padrão de ácido ascórbico.

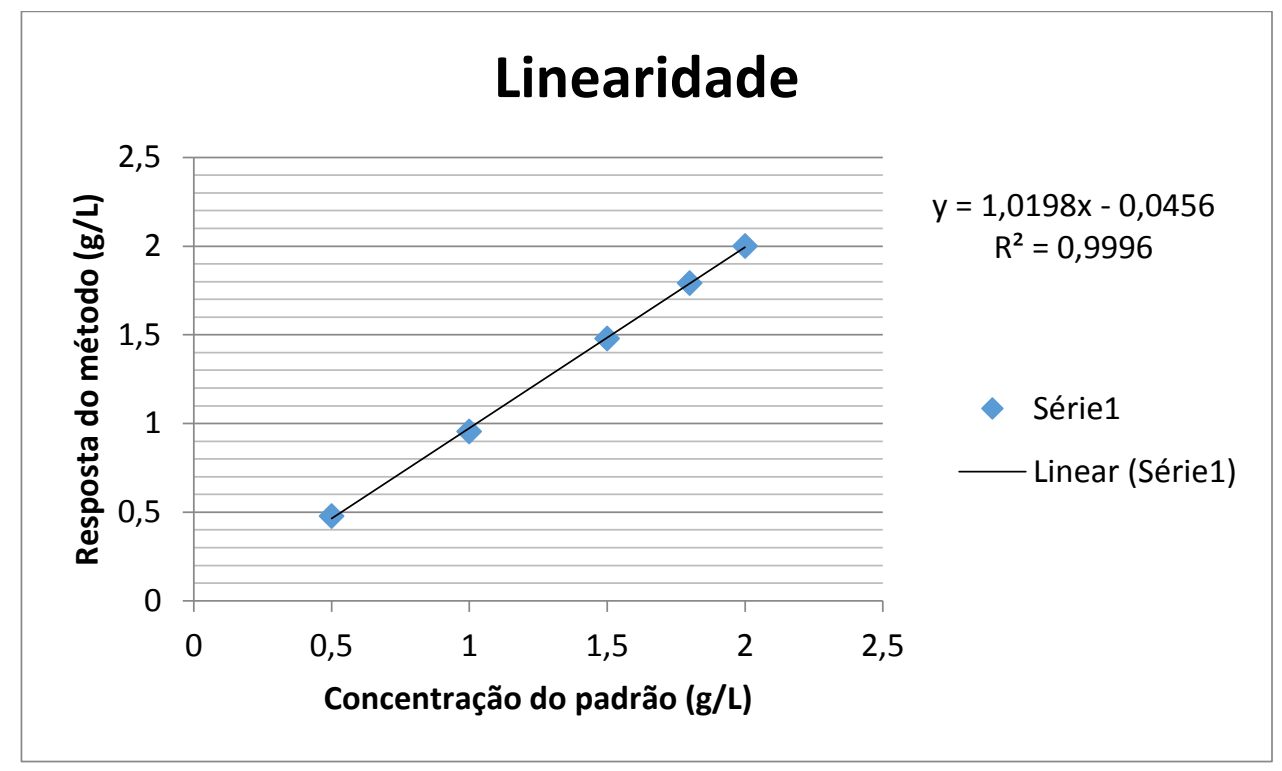

Seletividade do método:

Foi titutalado com um placebo, ou seja, todos os componentes de um comprimido efervescente exceto seu princípio atívo, no caso, o ácido ascórbico.

A primeira gota de iodo reage com o amido presente no erlenmeyer efetuando a mudança de coloração para azul escuro.

Incerteza de medição

Tabela 32: Tabela de incertezas

\begin{tabular}{ccc}
\hline Método/Variação & Amostra A & Amostra B \\
\hline Iodo 0,1 N & $0,38 \pm 0,17 \mathrm{~mol} / \mathrm{L}$ & $0,38 \pm 0,17 \mathrm{~mol} / \mathrm{L}$ \\
Iodo 0,1 N LabSynth ${ }^{\circledR}$ & $0,38 \pm 0,17 \mathrm{~mol} / \mathrm{L}$ & $0,32 \pm 0,18 \mathrm{~mol} / \mathrm{L}$ \\
\hline
\end{tabular}

\section{DISCUSSÃO}

Comparação entre as marcas:

O método mostrou-se mais exato e preciso quando analisado a amostra $\mathrm{B}$, por apresentar menor erro relativo e desvio padrão.

A hipótese nula, quando realizado o Teste $t$, não foi rejeitada pois $t_{\text {crit }}>t_{\text {calc. }} \mathrm{O}$ mesmo critério de rejeição é válido ao Teste $\mathrm{F}$, pois $\mathrm{F}_{\text {calc }}>\mathrm{F}_{\text {crit. }}$. 


\section{Revinter}

\section{Comparação entre diferentes volumes de amostra:}

É evidente que quanto maior o volume de amostra, tanto em A como em B titulado, mais exato e preciso é o método. É de conhecimento geral para aqueles que utilizam frequentemente a técnica de volumetria que quanto maior um volume de amostra para ser titulado melhor é a leitura do método.

Não foi rejeitado a hipótese nula quando realizada o Teste F para amostra A, já que o $F_{\text {crit }}>F_{\text {calc }}$, ou seja, não há diferença significativa entre os valores experimentais e é aceito a um nível de significÂncia de 95\%. Já para a amostra B foi rejeitado a hipótese nula, uma vez que $\mathrm{F}_{\text {calc }}>\mathrm{F}_{\text {crít. }}$

\section{Comparação entre diferentes concentrações de amido:}

Quando modificado o método e analisado a amostra A, não houve diferença significativas nas medidas de tendência central, dispersão e variabilidade. A análise de variância confirma esta teoria uma vez que o $\mathrm{F}_{\text {crit }}>\mathrm{F}_{\text {calc }}$, ou seja, não há reijeção da hipótese nula.

Quando modificado o método e analisado a amostra B, notou-se piora quando diminuiu a concentração do indicador. A análise de variância mostra que há a reijeição da hipótese nula e há significância nos valores experimentais.

\section{Comparação iodometria:}

Quando analisado a amostra A pelo método da iodometria, mostrou-se ser mais exato, no entanto, sem modificação significativa na precisão. A tabela da ANOVA mostrou que há diferença significativa entres os valores experimentais e com rejeição da hipótese nula. O Teste t mostrou que, também, há rejeição da hipótese nula, já que $t_{\text {calc }}>t_{\text {crít }}$

Já na análise da amostra $\mathrm{B}$ pelo método da iodometria, houve piora na exatidão e precisão dos resultados, uma vez que o erro relativo e o coeficiente de variação aumentaram significativamente. A tabela da ANOVA mostrou também que há diferença significativa entres os valores experimentais. Para o Teste t não houve rejeição da hipótese nula.

\section{Comparação com iodo 0,2 $\mathrm{N}$ :}




\section{Revinter}

Assim como dito anteriormente, para a amostra A o método mostrou-se mais exato e preciso, já para a amostra $\mathrm{B}$ menos exato e menos preciso.

Para ambos os casos, a tabela da ANOVA, através do Teste F, mostrou que não há a rejeição das hipóteses nulas.

E o Teste t, para a amostra A houve reijeição da hipótese nula e para amostra B não.

\section{Comparação com iodo de outra procedência:}

Para a amostra A, a varição mostrou-se ser mais precisa, no entanto não houve alterações na exatidão do método. Para o Teste F não houve reijeição da hipótese nula, mostrando que não há diferença significativa entre os resultados. No entanto, para o Teste $t$ houve rejeição da hipótese nula.

Para a amostra B, o método mostrou-ser ser menos exato e preciso. O Teste $\mathrm{F}$ mostrou que há diferença significativa com rejeição da hipótese nula, no entanto, o Teste t mostrou que não há rejeição da hipótese nula e portanto sem diferença significativa entre as médias.

Comparação com solução de tiossulfato de sódio de outra procedência (comparação entre os métodos iodométricos):

Para ambas amostras, a modificação mostrou ser menos exata, no entanto mais precisa, ainda que haja uma diferença baixa. A análise de variância mostrou que não há diferença significativa entre os valores, portanto sem rejeição da hipótese nula. $\mathrm{O}$ Teste $\mathrm{t}$ conclui que se deve rejeitar a hipótese nula pois, há diferneça significativa entre os valores experimentais a um nível de significância de $95 \%$.

\section{Comparação entre os analistas:}

A maior relevância para este tipo de comparação é a análise de variância. Para a amostra A, não há diferença significativa entre os valores experimentais. Já para a amostra $\mathrm{B}$, há diferença signficativa, pois $\mathrm{F}_{\text {calc }}>\mathrm{F}_{\text {crít, }}$, portanto rejeita-se a hipótese nula.

\section{Comparação entre diferentes concentrações de amostras:}

Para este tipo de parâmetro, leva-se em consideração a teoria da regressão linear, definida por $\mathrm{y}=\mathrm{ax}+\mathrm{b}$ e o coeficiente de regressão linear " $\mathrm{r}$ ". 


\section{Revinter}

O método mostrou ser linear por apresentar coeficiente linear 0,9996, portanto aceito segundo os critérios de aceitabilidade que exigem proximidade a 1 , ou seja, em diferças concentrações o método efetua uma regressão linear.

\section{Seletividade}

Como a primeira gota de iodo foi suficiente para a mudança de cor do titulado, o método é totalmente seletivo, uma vez que se sabe que a primeira gota em excesso de iodo reage com o amido presente no titulado e muda-se a coloração para azul excuro.

\section{Incerteza de medição}

Apesar de reduzir a quantidade de fontes de incertezas, quandou mudou a procedência de solução padrão de iodo, não foi o suficiente para mudar significativamente a incerteza de medição.

\section{CONCLUSÃO}

Portanto, o método avalidado não respondeu de maneira correta para as modificações, já que praticamente todos os parâmetros houve diferença significativa ou entre as amostras ou entre os próprios parâmetros.

\section{REFERÊNCIAS BIBLIOGRÁFICAS}

AREDS- Age-Related Eye Disease Study Research Group. A randomized, placebo controlled, clinical trial of high-dose supplementation with vitamins $\mathrm{C}$ and $\mathrm{E}$, beta carotene, and zinc for age-related macular degeneration and vision loss: AREDS report no. 8. Arch Ophthalmol 2001 Oct;119(10):1417-36.

ASSOCIAÇÃO BRASILEIRA DE NORMAS TÉCNICAS. NBR 11589: Preparação, padronização e estocagem de soluções para análise química. Rio de Janeiro: 1990.

BACCAN, N.; DE ANDRADE, J. C.; GODINHO, O. E. S.; BARONE, J. S. Química analítica quantitativa elementar. São Paulo: Edgar Blücher; Campinas: Universidade Estadual de Campinas, 1979.

BLUMBERG, J. Nutrient requirements of the healthy elderly: should there be specific RDAs? Nutrition Reviews, New York, v.52, n.8, p.15S-18S, 1994. Supplement. 


\section{Revinter}

COUTO, M. A. L, CANNIATTI-BRAZACA, S. G. Quantificação de vitamina C e capacidade antioxidante de variedades cítricas. Ciência e Tecnologia de Alimentos, v.30, 2010.

ESCOTT_STUMP, S.; KRAUSE.; MAHAN, L.K.; Alimentos, nutrição e dietoterapia [tradução Natalia Rodrigues Pereira...et al.]. Rio de Janeiro: Elsevier, 2010; p. 95-96 e 100.

EURACHEM/CITAC. Determinando a incerteza na Medição Analítica. 2a ed.

FUCHS F. D.; WANNMACHER; L. Farmacologia Clínica: Fundamentos da Terapêutica Racional. 4a ed. Rio de Janeiro: Guanabara Koogan; 2010.

GUILLAND, J.C., LEQUEU, B. As vitaminas do nutriente ao medicamento. São Paulo : Santos, 1995. 375p.

LAJOLO, F. M.; PFRIMER, K. Nutrição e envelhecimento saudável. São Paulo: ILSI Brasil - International Life Sciences Institute do Brasil, v. 6, p. 24-26, 2016.

NEDER, H. D. Curso de estatística aplicada. Instituto de economia, Universidade federal de Uberlândia. 20??

RUSSO, A.; SANT'ANNA, M. Uso racional da Vitamina C (Ácido Ascórbico). Cebrim Informa, 2013.

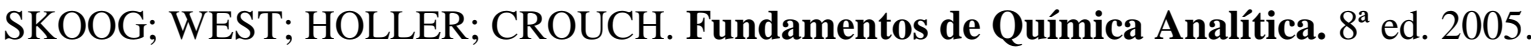

TEIXEIRA NETO, F.; Nutrição clínica Rio de Janeiro: Guanabara Koogan, 2009; p. 76, $128-129$ e 288.

VIEIRA, S. Estatística para a qualidade. $2^{\mathrm{a}}$ ed. Rio de Janeiro: Elsevier, 2012.

\footnotetext{
¡ Técnico em Química pela ETEC Tiquatira; Graduando em Farmácia pela Universidade São Judas Tadeu.

ii Técnico em Química pela ETEC Tiquatira.

iii Técnico em Química pela ETEC Tiquatira.

iv Técnico em Química pela ETEC Tiquatira; Graduando em Engenharia Ambiental pela UNESP.

v Técnico em Química pela ETEC Tiquatira.

vi Graduação em Química pela Universidade Castelo branco e docente da ETEC Tiquatira.

vii Graduação em Farmácia pela Universidade São Judas Tadeu; Mestrado em Toxicologia e Análises Toxicológicas pela Universidade de São Paulo; Doutorado em Patologia Experimental e Comparada pela Universidade de São Paulo.
} 